\title{
Presentation of Tuberculous Meningitis Patients : Study of 30 Cases
}

\author{
Sarkar $\mathrm{DN}^{1}$, Hossain $\mathrm{MI}^{2}$, Shoab $\mathrm{AKM}^{3}$, Quraishi $\mathrm{FA}^{4}$
}

\begin{abstract}
Tuberculosis is creating immense adverse social and economic burden in Bangladesh. Tuberculous Meningitis results from the haematogenous spread of primary or postprimary pulmonary disease or from the rupture of a subependymal tubercle into the subarachnoid space. Infection of the CNS is one of the most devastating clinical manifestations of tuberculosis
\end{abstract}

This study aimed to see the presentation of tuberculous meningitis patients in tertiary level hospital of Bangladesh. This cross sectional study was carried out in the Department of Neurology, SSMC, Dhaka from June 2011 to July 2012.

In the present study, thirty meningitis patients were enrolled, among the subjects $11.7 \%$ were aged below 20 years, $53.3 \%$ were aged between 20 - 39 years, $16.7 \%$ were aged between 40 - 49 years and $18.3 \%$ were aged above 50 years. Regarding sex $56.7 \%$ were male and $43.3 \%$ were female. Most prevalent symptom was fever (91.7\%). Among others, headache (70\%), altered consciousness (45\%), vomiting (43.3\%) and neck stiffness (28.9\%) were notable. The distribution of the study subjects by physical sign 55.0\% had anemia, 95.0\% had neck rigidity, 10.0\% had cranial nerve palsy, $46.7 \%$ had kernig's sign and $20.0 \%$ had long tract sign. Regarding GCS score $10.0 \%$ had GCS score less than $8,18.3 \%$ had between $9-12$ and $71.7 \%$ had over $>$ 12.The Laboratory finding among the study subjects 3.3\% showed positive gram stain and $43.7 \%$ showed MT over 10 $\mathrm{mm}$. In response to treatment $93.3 \%$ responded to anti $T B$ out of 30 TBM subjects.

This study demonstrated that presentation of tubercular meningitis is different in different patient.TBM is the severe form of extra-pulmonary tuberculosis occurring in 7.0$12.0 \%$ of TB patients in developing countries with high rate of mortality due to delay in diagnosis and proper treatment.

1. Devendra Nath Sakar MCPS, MD, FCCP, FRSM, FACP Associate Professor, Department of Medicine Rangpur Medical College \& Hospital, Rangpur

2. Corresponding Author: Md. Ismail Hossain MBBS Registrar, Department of Medicine Rangpur Medical College \& Hospital, Rangpur email-drrussel27@gmail.com

3. A K M Shoab MCPS, MD Junior Consultant of Medicine UHC, Rajnagar, Moulvibazar, Bangladesh

4. Firoz Ahmed Quraishi FCPS, MD Professor of Neurology National Institute of Neuroscience, Dhaka, Bangladesh

\section{Introduction}

Tuberculosis is one of the leading causes of mortality and morbidity in developing countries. The WHO reports puts to the record that globally, approximately 16 million people are suffering from active TB with an estimated 8.5 million developing active TB each year, resulting in approximately 2 million deaths ${ }^{1}$. Tuberculous meningitis (TBM) is a common infectious disease of the central nervous system in developing countries. Early diagnosis and treatment with chemotherapy and active management of the complications are of great importance to prevent the irreversible neurologic sequele and death. Delay in diagnosis and so in the start of effective treatment results in poor prognosis and sequalae in up to $25 \%$ of cases $^{2}$. A definitive diagnosis of tuberculous meningitis depends on identifying Mycobacterium tuberculosis in the cerebrospinal fluid (CSF) by direct staining or culture. However, the diagnostic yield of CSF smears and cultures has been very low $^{3}$, and mycobacterial culture may take up to 6 weeks to yield results. Therefore, the diagnosis of tuberculous meningitis depends on the clinical manifestations of subacute to chronic meningitis with lymphocytic CSF and low CSF glucose levels. However, other forms of meningitis may mimic tuberculous meningitis. Certain patients with tuberculous meningitis may have CSF findings resembling aseptic meningitis. Several tests for the rapid diagnosis of tuberculous meningitis have been developed; all are based on examination of the CSF. These tests are considered indirect tests (usually measuring a product of the host response to his infection, such as adenosine deaminase(ADA), the radioactive bromide partition test and antibodies to the mycobacterial antigen) and direct tests usually measuring a product of the infecting organism, such as 3-(2'-ketohexyl) indoline, detecting of tuberculostearic acid (a component of the cell wall of M. tuberculosis), mycobacterial antigens or fragments of mycobacterial DNA by polymerase chain reaction ${ }^{4}$.

\section{Materials and Methods}

It is a cross sectional study. Thirty cases of tuberculous meningitis were studied. All eligible subjects as per inclusion and exclusion criteria coming to the Department of Neurology and Medicine, Sir Salimullah Medical College \& Mitford Hospital,Dhaka Medical College Hospital and Bangabandhu Sheikh Mujib Medical University from July 2011 to June 2012 were included till desired sample size was reached. The laboratory works were performed in the department of microbiology \& immunology, BSMMU and Sir Salimullah Medical College, Dhaka. 


\section{Statistical Analysis}

Data was analyzed using SPSS 16 statistical package. A descriptive analysis was done on all variables to obtain a frequency distribution. The mean $\pm \mathrm{SD}$ and ranges were calculated for quantitative variables. Continuous variables were compared by the Student t test. Proportions were analyzed with the chi-square test or 2 - tailed Fisher's exact test as appropriate. A P value of 0.05 or less was considered statistically significant.

\section{Results}

Table-I shows the distribution of the study subjects by age and sex. Among the subjects $11.7 \%$ were aged below 20 years, $53.3 \%$ were aged between 20 - 39 years, $16.7 \%$ were aged between 40 - 49 years and $18.3 \%$ were aged above 50 years. Regarding sex $56.7 \%$ were male and $43.3 \%$ were female.

Table I: Distribution of the respondents by age and sex

\begin{tabular}{lcc}
\hline Age in years & Frequency & Percentage \\
$15-20$ & 4 & 11.7 \\
$20-39$ & 16 & 53.3 \\
$40-49$ & 5 & 16.7 \\
$>=50$ & 5 & 18.3 \\
Sex & & \\
Male & 17 & 56.7 \\
Female & 13 & 43.3 \\
\hline
\end{tabular}

Figure-1 depicts the distribution of the study subjects by presenting symptoms. Among them most prevalent symptom was fever $(91.7 \%)$. Among others, headache (70\%), altered consciousness (45\%), vomiting (43.3\%) and neck stiffness $(28.9 \%)$ were notable.

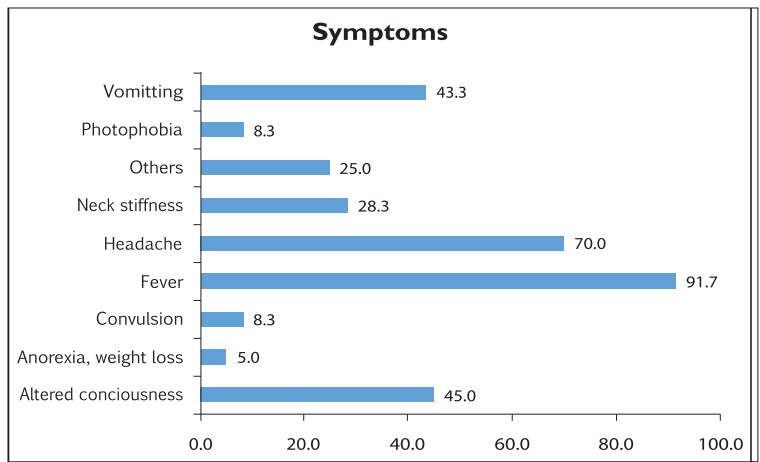

Figure-1: Distribution of the study subjects by presenting symptoms

Table II shows the distribution of the study subjects by physical sign. Among the study subjects $55.0 \%$ had anemia, $95.0 \%$ had neck rigidity, $10.0 \%$ had cranial nerve palsy, $46.7 \%$ had kernig's sign and $20.0 \%$ had long tract sign.
Table-II: Distribution of the study subjects by physical sign

\begin{tabular}{lcc}
\hline Physical sign & Frequency & Percentage \\
Anemia & 17 & 55.0 \\
Neck Rigidity & 29 & 95.0 \\
Cranial Nerve Palsy & 3 & 10.0 \\
Kernig's sign & 14 & 46.7 \\
Long tract sign & 6 & 20.0 \\
\hline
\end{tabular}

Table III shows the distribution of the study subjects by fundoscopic examination finding, $83.3 \%$ had normal eye, $3.3 \%$ had optic atrophy and $13.3 \%$ had papilloedema.

Table-III: Distribution of the study subjects by fundoscopic examination finding

\begin{tabular}{lcc}
\hline Findings & Frequency & Percentage \\
Normal & 25 & 83.3 \\
Optic atrophy & 1 & 3.3 \\
Papilloedema & 4 & 13.3 \\
Total & 30 & 100.0 \\
\hline
\end{tabular}

Figure 2 shows the distribution of the study subjects by GCS score. Among the study subjects $10.0 \%$ had GCS score less than $8,18.3 \%$ had between $9-12$ and $71.7 \%$ had over $>12$.

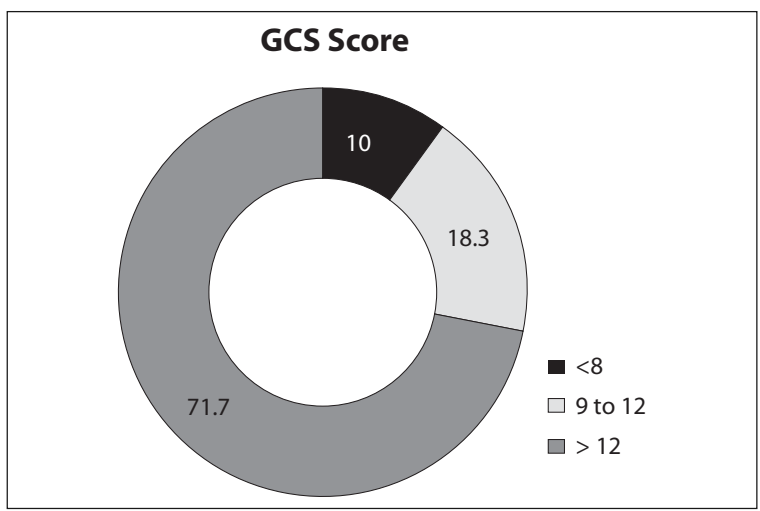

Figure-2: Distribution of the study subjects by GCS score

Table IV shows the biochemical parameters of CSF in various meningitis. In TBM, sugar was low $2.6 \pm 1.2(0.8-$ $6.5)$, protein was high $212.3 \pm 275$. (137-1440), total leucocytes count was high 484.7 1317 (5-6400) and lymphocytic pleocytosis $72.08 \pm 31.2(10-100)$. In bacterial meningitis, sugar was low $2.606 \pm 1.2(0.4-4.5)$, protein was high $142.1 \pm 99.7$ (66-388), total cell count was high $192.4 \pm 199.9(5-840)$ and polymorph was $68 \% 68.75 \pm 37.5$ (0-95).In viral meningitis, sugar was normal 4.01 \pm 1.1 (2.1$6.6)$, protein was mildly high $73.8 \pm 55.2(2-226)$, cell count was high $27.24 \pm 48.3(0-180)$ mostly lymphocytes $88.24 \pm 23.4$ (10-100).

Table $\mathrm{v}$ shows the distribution of the Laboratory finding. Among the study subjects $3.3 \%$ showed positive gram stain. And $43.7 \%$ showed MT over $10 \mathrm{~mm}$. 
Table -IV: Biochemical parameters of CSF in various Meningitis

\begin{tabular}{lclllll}
\hline & \multicolumn{2}{c}{ A. Tuberculous } & \multicolumn{2}{c}{ B. Bacterial } & \multicolumn{2}{c}{ C. Viral } \\
\hline & Mean \pm SD & Range & Mean \pm SD & Range & Mean \pm SD & Range \\
Sugar $(\mathrm{mmol} / \mathrm{L})$ & $2.6 \pm 1.2$ & $0.8-6.5$ & $2.606 \pm 1.2$ & $0.4-4.5$ & $4.01 \pm 1.1$ & $2.1-6.6$ \\
Protein $(\mathrm{mg} / \mathrm{dl})$ & $212.3 \pm 275.1$ & $37-1440$ & $142.1 \pm 99.7$ & $66-388$ & $73.8 \pm 55.2$ & $21-226$ \\
TC $(/ \mathrm{cmm})$ & $484.7 \pm 1317$ & $5-6400$ & $192.4 \pm 199.9$ & $5-840$ & $27.24 \pm 48.3$ & $0-180$ \\
lymphocyte $(\%)$ & $72.08 \pm 31.2$ & $10-100$ & $31.25 \pm 36.8$ & $5-100$ & $88.24 \pm 23.4$ & $10-100$ \\
Polymorph $(\%)$ & $28.12 \pm 31.6$ & $0-95$ & $68.75 \pm 37.5$ & $0-95$ & $12.1 \pm 6.47$ & $0-44$ \\
\hline
\end{tabular}

Table -V: Distribution of the Laboratory findings

\begin{tabular}{lcc}
\hline & Frequency & Percentage \\
Gram Staining $(\mathrm{n}=30)$ & 1 & 3.3 \\
Positive & 29 & 96.7 \\
Negative & & \\
MT $(\mathrm{n}=30)$ & 17 & 56.3 \\
$<10 \mathrm{~mm}$ & 13 & 43.7 \\
$>=10 \mathrm{~mm}$ & & \\
\hline
\end{tabular}

Table VI shows the distribution of the study subjects by response to treatment. Among the study participants $93.3 \%$ responded to anti TB out of $30 \mathrm{TBM}$ subjects.

Table VI: Distribution of the study subjects by response to treatment

\begin{tabular}{lcc}
\hline & Frequency & Percentage \\
Response to Anti-TB $(\mathrm{n}=30)$ & & \\
Yes & 28 & 93.3 \\
No & 02 & 6.7 \\
\hline
\end{tabular}

\section{Discussion}

The present study was done in 30 cases (TBM).However,among the 30 TBM cases, one case was from NTBM group as intially diagnosed as pyogenic meningitis but latter on due to lack of antibiotic response and repeat CSF showed featues of TBM, it was included as TBM.Similarly two clinically suspected TBM cases were lastly diagnosed as meningitis due to lymphoma and another as Wilson's disease. So these two cases were excluded from the study based on exclusion criteria. The peak incidence in the present study was found in young adults in the age group of 20-39years $(53.3 \%)$. It is similar to another study where observed the peak incidence was $43 \%$. According to the present study, the incidence in males was $56.7 \%$ and in females $43.3 \%$. The incidence in both males and females is consistent with the study done by Gambhir ${ }^{6}$. The reasons for the high occurance of infection in male might be due to the fact that being the main earning member of the family; they have to go outside and thus are more exposed and more chances of getting infections. Moreover females are reluctant to come to the hospital for treatment.

In the present study, history of fever is present in most of the cases $(91.7 \%)$. It was low-grade, more in the evening, associated with night sweats. In other study the incidence of fever was $87 \%$ and $58.9 \%{ }^{7,8}$. Fever was absent in about
$10 \%$ cases; litrature told that fever can be absent in upto $25 \%$ of patients ${ }^{9}$. Seizures of generalized tonic and clonic type were noted in $8.3 \%$ of the cases of both TBM and NTBM groups. In one study, the incidence of seizures was $12.1 \%{ }^{8}$. The signs of meningeal irritation were present as neck rigidity in 95\%, Kernig's sign in $46.7 \%$.In another study, neck rigidity was $54 \%$ and Kernig's sign was $40 \%$ cases $^{10}$ and on the other hand, neck rigidity was $100 \%$ in another study in neurology text book, the overall meningeal signs is mentioned as $70 \%^{11}$. The present study revealed that cranial nerve palsies were observed in $10 \%$ of the cases, which is consistent with the other study and that was observed in $15.4 \%$ cases.However, it was $50 \%^{10}$ of cases also observed.The commonnest was 6 th $50 \%(3 / 6)$, then 7 th $16.6 \%(1 / 6$ case $)$ and one was third cranial nerve palsy ; another one case was with multiple cranial nerve palsy (vi,vii,ix,x,xi,xii). In one study it was found 6thnerve palsy in $7.2 \%$ cases $^{8}$ and also reported $3.2 \%$ cases with isolated facial nerve palsies ${ }^{11}$.In the present study, the incidence of papilloedema was $13.3 \%$ and optic atrophy $3.3 \%$ of cases and all are in TBM group not the NTBM. Other study observed papilloedema in $16.1 \%$ of cases $^{11}$. In the presenet study, limb weakness was noted in $20 \%$ cases, higher rate of observation was also found and the limb weakness was $37.9 \%$ cases $^{11}$ but literaure review shows focal neurological findings was in $16-18 \%^{12}$ that is more similar to present study. Similarly MT test shows higher rate of positivty in TBM patients and it was positive in $43.7 \%$ cases.MT positive found in $50 \%$ cases of $\mathrm{TBM}^{12}$.

The biochemical parameters of CSF in various meningitis showed some disimilarity between TBM and pyogenic meningitis for example CSF total cell count was higher in TBM than pyogenic meningitis. It was possibly due to getting partial treatment of pyogenic meningitis before coming to tertiarry level hospital.Several case series have established CSF staining sensitivities of $<20 \%{ }^{13,14}$. However in the present study CSF ZN staning for AFB of all cases were negative. MTB culture studies in several case series established CSF culture sensitivities of 25 to $70 \%{ }^{15,16}$ and in one study shows the sensitivity of AFB culture is $40 \%{ }^{17}$. In the present study $36.6 \%(11 / 30)$ cases were culture positive.

30 patients got anti-TB therapy among them 28 patients responded and 2 cases died. Both of them were in stage iii with complications.Among the the 28 caes, 27 cases got Cat-I treatment, only one got Cat-II regimen because of 
recurrent TBM. 3 cases got sequlae like blindness (1 unilateral \&1 bilateral) and paraplegia due to (TBRM) tuberculous myeloradiculitis( 1 case). 15 cases(50\%) had gone for brain imaging due to clinical suspicion of raised ICP with focal deficit and most of them got radiological abnormality such as tuberculoma(1case), nonobstucting hydrocephalus ( 8 cases), ischemic changes ( 2 cases),contrast enhanced lesion-ring/meningeal enhanement-(3 cases). Among the 15 cases, 5 cases got surgical intervention (ventriculoperitoneal shunt) due to raised ICP.

TBM is the severe form of extrapulmonary tuberculosis occurring in $7.0-12.0 \%$ of $\mathrm{TB}$ patients in developing countries with high rate of mortality due to delay in diagnosis and proper treatment.In the absence of an early diagnosis and treatment, tuberculous meningitis is characterized by high mortality $(20-50 \%)$ and morbidity (20-30\%). The cytological and biochemical analysis of cerebrospinal fluid is the cornerstone for diagnosis but there are diagnostic difficulties many a times in differentiating tuberculous meningitis from nontubeculous meningitis.

\section{References}

1. WHO reports, Global TB control, surveillance, planning, financing; communicable diseases, WHO, Geneva 2003;18.

2. Garcia-Monco JC, Marra CM, editor. CNS Tuberculosis. Neurologic Clinics.1999;17:737-760.

3. Chotmongkol V, Jitpimolmard S and Thavornpitak Y. 'Corticosteroid in tuberculous meningitis', J Med Assoc Thai.1996;79:83-90.

4. Zuger A, Lowy FD.Tuberculosis of the central nervous system. In: Scheld WM, Whitley RJ,Durack DT, eds. Infections of the central nervous system. New York: Raven Press, 1991;425-56.

5. Ramkishna, 2006, Adenosine deaminase acitivity in cerebrospinal fluid for diagnosis of tubrculosis meningitis, www.scribd.com/doc/52584960/ada in-tbmeningitis.
6. Gambhir IS, Mehta M, Singh DS and Khanna HD. 'Evaluation of CSF-adenosine deaminase activity in tubercular meningitis', J Assoc physician India.1999;47:192-194.

7. Khatua SP. 'Tuberculous meningitis in children: Analysis of 231 cases', J.Indian Med Ass.1961;37:332.

8. Virmani Vimla,Venkataraman S, Sarasavani, V Rao, and Ahuja GK, 'Clinical spectrum of neurotuberculosis in adults', Jr Asso Phys Ind. 1980;28:431-437.

9. Tarakad S Ramachandran, Tuberculous Meningitis; Chief Editor: Karen L Roos; Medscape; http://emedicine.medscape.com/article/2011;1166190

10. Khatua SP. 'Tuberculous meningitis in children: Analysis of 231 cases', J.Indian Med Ass.1961;37:332.

11. Venkataraman S, Sarasavani, V Rao, Ahuja GK and VimlaVirmani, 'Clinical spectrum of neurotuberculosis in adults', JrAssoPhys Ind.1980;28:431-437.

12. Ashok Verma. Infections of the nervous system.In:WG Bradely, RB Darott,GM Fenidel,J Jancovic(eds).Neurology in Clinical Practice;5th edn, New York,Elsevier:2008;1420.

13. Tofte RW, Peterson PK, Kim Y, 'Opsonic activity in normal human cerebrospinal fluid for selected bacterial species', Infect Immun.1979;26:1093-8.

14. Sutlas P N, A Unal, H Forta, S Senol, and D Kirbas, 'Tuberculous meningitis in adults: review of 61 cases', Infection.2003;31:387-391.

15. Kent S J, S M Crowe, A Yung, C R Lucas and A M Mijch. 'Tuberculous meningitis: a 30-year review', Clin. Infect. Dis.1993;17:987-994.

16. Naughten E, A M Weindling, R Newton, and B D Bower. 'Tuberculous meningitis in children. Recent experience in two English centres', Lancet ii.1981;973-975.

17. Ogawa S K, M A Smith, D J Brennessel and F D Lowy,1987. 'Tuberculous meningitis in an urban medical center', Medicine (Baltimore.)1987;66:317-326. 